Document downloaded from:

http://hdl.handle.net/10251/53150

This paper must be cited as:

Selga, J.; Rodríguez Pérez, AM.; Orellana, M.; Boria Esbert, VE.; Martín, F. (2014). Automated synthesis of transmission lines loaded with complementary split ring resonators (CSRRs) and open complementary split ring resonators (OCSRRs) through aggressive space mapping (ASM). Applied Physics A. 117(2):557-565. doi:10.1007/s00339-014-8703-x.

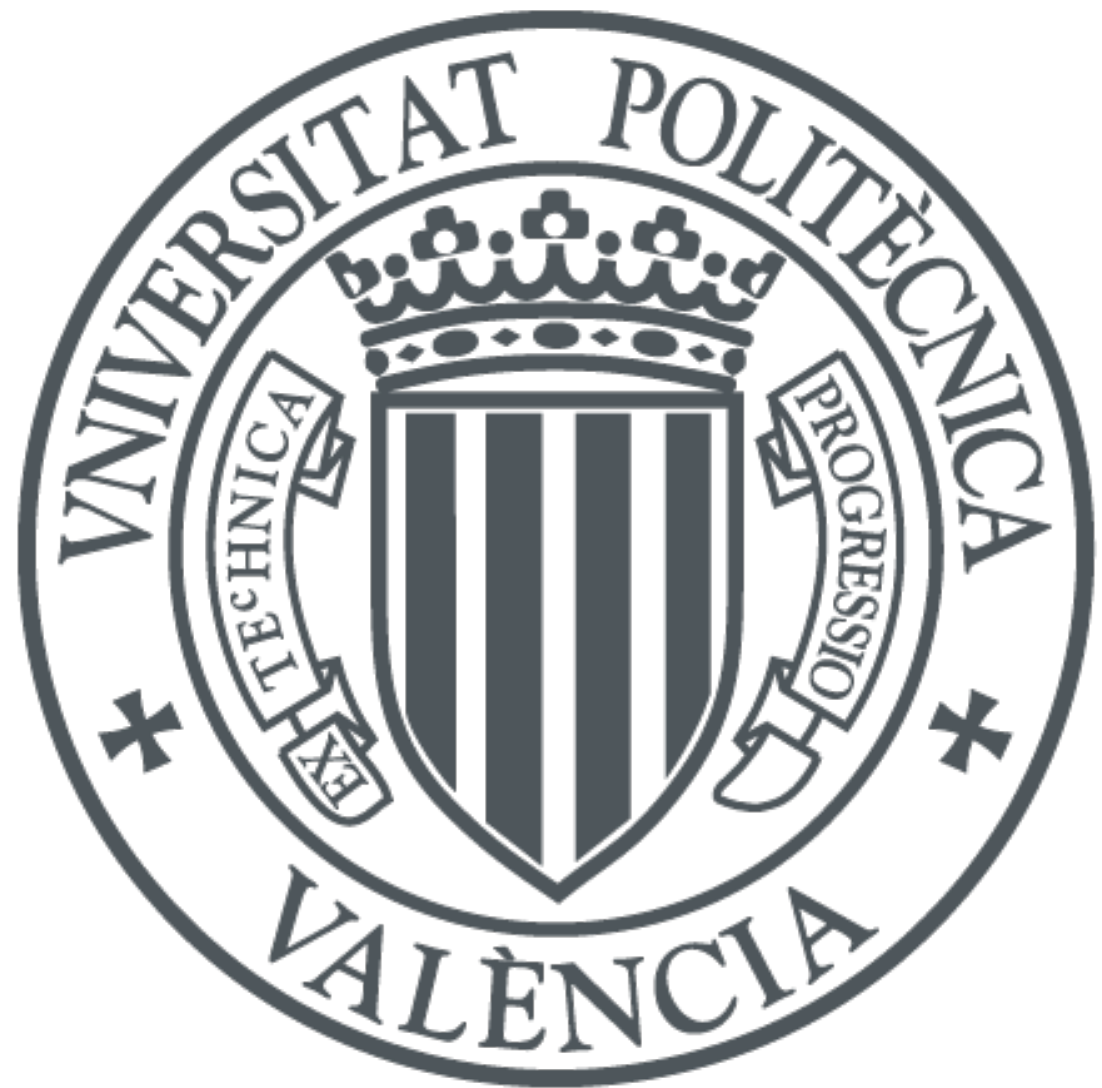

The final publication is available at

http://dx.doi.org/10.1007/s00339-014-8703-x

Copyright Springer Verlag

Additional Information 


\title{
Automated Synthesis of Transmission Lines Loaded with Complementary Split Ring Resonators (CSRRs) and Open Complementary Split Ring Resonators (OCSRRs) through Aggressive Space Mapping (ASM)
}

\author{
Jordi Selga ${ }^{* 1}$, Ana Rodríguez ${ }^{2}$, Marco Orellana ${ }^{1}$, Vicente Boria ${ }^{2}$, Ferran Martín ${ }^{1}$ \\ ${ }^{1}$ GEMMA/CIMITEC, Departament d'Enginyeria Electrònica, Universitat Autònoma de Barcelona, 08193 Bellaterra, Spain \\ ${ }^{2}$ Departamento de Comunicaciones-iTEAM, Universidad Politécnica de Valencia, 46022 Valencia, Spain \\ *corresponding author, E-mail: Jordi. Selga@uab.cat
}

\begin{abstract}
This paper is focused on the application of space mapping optimization to the automated synthesis of transmission lines loaded with complementary split ring resonators (CSRRs) and open complementary split ring resonators (OCSRRs). These structures are of interest for the implementation of resonant-type metamaterial transmission lines, and for the design of planar microwave circuits based on such complementary resonators. The paper presents a method to generate the layouts of CSRR- and OCSRRloaded microstrip lines from the elements of their equivalent circuit models. Using the so-called aggressive space mapping (ASM), a specific implementation that uses quasi-Newton type iteration, we have developed synthesis algorithms that are able to provide the topology of these CSRR- and OCSRR-loaded lines in few steps. The most relevant aspect, however, is that this synthesis process is completely automatic, i.e., it does not require any action from the designers, other than initiate the algorithm. Moreover, this technique can be translated to other electrically small planar elements described by lumped element equivalent circuit models.
\end{abstract}

\section{Introduction}

Metamaterial transmission lines have attracted the attention of $\mathrm{RF} / \mathrm{microwave}$ engineers in the last years due to their enhanced design flexibility as compared to ordinary lines [1-4]. Such lines are implemented by loading a host line with reactive elements (inductors, capacitors, resonators, or a combination of them). Thanks to the presence of such additional elements, metamaterial transmission lines exhibit functionalities not easily achievable in conventional lines, mostly related to the fact that their dispersion can be engineered or tailored to satisfy certain requirements. This extra controllability of the dispersion diagram (and characteristic impedance as well) has open the door to the design and implementation of enhanced bandwidth components [5-7], multiband and multifunctional devices [8-10], compact filters and diplexers [11,12], backfire-toendfire leaky wave antennas $[13,14]$, etc.
One of the drawbacks in the design of microwave circuits based on metamaterial transmission lines is related to their synthesis. This is due to the presence of the loading elements and their interaction with the host line. There are two main approaches for the implementation of metamaterial transmission lines: (i) the CL-loaded approach, where a host line is loaded with series capacitances and shunt inductances [15-17], and (ii) the resonant-type approach, where the host line is loaded with electrically small resonant elements, typically (although not exclusively) split ring resonators (SRRs) or complementary split ring resonators (CSRRs), and additional reactive elements [18,19]. This work is focused on the second approach, specifically on the automated synthesis of metamaterial transmission lines based on CSRRs. Moreover, we will also consider the synthesis of microstrip lines loaded with open complementary split ring resonators (OCSRRs), of interest for the implementation of metamaterial transmission lines (in combination with OSRRs [20]) and bandpass filters [21].

\section{CSRR- and OCSRR-loaded microstrip lines}

The considered structures are microstrip lines loaded with CSRRs etched in the ground plane (Fig. 1a), and microstrip lines loaded with OCSRRs connected to ground through via holes (Fig. 2a). CSRR-loaded lines are described by the lumped element circuit depicted in Fig. 1(b), where the CSRR is modeled by the $L_{c}-C_{c}$ resonant tank, $C$ models the coupling between the line and the resonator, and $L$ accounts for the line inductance [22]. According to the circuit model, CSRR-loaded lines exhibit a stopband behavior (there is a transmission zero at the frequency where the shunt branch shorts to ground). This behavior can also be attributed (in a line loaded with an array of CSRRs) to the negative effective permittivity and large positive effective permittivity above and below, respectively, the resonance frequency of the CSRRs. It is also well-known that by adding series capacitive gaps to CSRR-loaded lines, the line exhibits also a negative effective permeability in a certain band, and the structure presents a composite right/left handed (CRLH) behavior [23]. Both types of lines CSRR- 
and CSRR/gap-loaded lines have been applied to the design of many circuits, being the synthesis, in general, a complex task. This has motivated our research activity towards obtaining algorithms able to help the designers in finding the appropriate topologies to satisfy the requirements or specifications.

OCSRR-loaded microstrip lines are described by the circuit model depicted in Fig. 2(b), where the effect of the access lines has been ignored. It has been demonstrated that the structure of Fig. 2(a) is accurately described by this model [20,24], where the inductance $L_{s h}$ is included to account for the strip (of width $e$ ) present between the inner metallic region of the OCSRR and the access lines (or ports). In combination with OSRR (described by a series resonator in a first order approximation [20]), OCSRR are of interest for the implementation of CRLH lines and filters. It is also possible to implement bandpass filters by coupling OCSRRs through admittance inverters in microstrip technology [25].
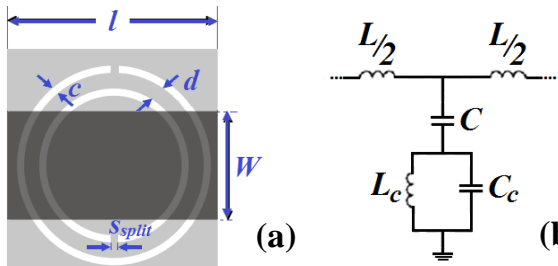

Figure 1: Typical topology (a) and circuit model (b) of a CSRR-loaded microstrip line (the ground plane is indicated in light grey).
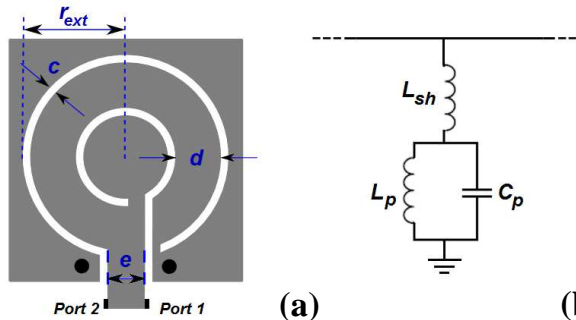

(a)

(b)

Figure 2: Typical topology (a) and circuit model (b) of a OCSRR-loaded microstrip line. Vias are indicated with black dots.

In the next section, it is briefly explained how CSRRand OCSRR-loaded microstrip lines can be synthesized with the use of the so-called aggressive space mapping (ASM) algorithm.

\section{Synthesis of CSRR and OCSRR loaded lines by means of ASM}

In this section, a general formulation of ASM is first given, and then it is applied to obtain the automated synthesis of the considered structures.

\subsection{Aggressive space mapping (ASM)}

Space mapping is a technique extensively used for the synthesis of microwave devices [26-28]. It makes use of two simulation spaces: in the optimization space, $\mathbf{X}_{c}$, the variables are linked to a coarse model, which is simple and computationally efficient, although not accurate; in the validation space, $\mathbf{X}_{f}$, the variables are linked to a fine model, typically more complex and CPU intensive, but significantly more precise. In each space, a vector containing the different model parameters is defined. Such vectors are designated as $\mathbf{x}_{f}$ and $\mathbf{x}_{c}$ for the fine and coarse model parameters, respectively, and their responses are denoted as $\mathbf{R}_{f}\left(\mathbf{x}_{f}\right)$ and $\mathbf{R}_{c}\left(\mathbf{x}_{c}\right)$. In planar microwave circuits, the vectors $\mathbf{x}_{f}$ and $\mathbf{x}_{c}$ typically contain a set of geometric and circuit parameters, respectively, whereas the model responses are related to the evaluation of the device behaviour, e.g., a scattering parameter, such as $S_{11}$ or $S_{21}$, computed in a certain frequency range.

ASM [27] is an advanced space mapping optimization technique that uses a quasi-Newton type iteration to find the target solution. The goal in ASM is to solve the following set of non-linear equations

$$
\mathbf{f}\left(\mathbf{x}_{f}\right)=\mathbf{P}\left(\mathbf{x}_{f}\right)-\mathbf{x}_{c}^{*}=0
$$

where $\mathbf{x}_{c}{ }^{*}$ is the vector that contains the target circuit elements, and $\mathbf{P}\left(\mathbf{x}_{f}\right)$ is a function that gives the extracted circuit parameters from the electromagnetic response of the layout. For a better understanding of the iterative optimization process, a superscript is added to the notation that actually indicates the iteration number. Hence, let us assume that $\mathbf{x}_{f}^{(j)}$ is the $j$-th approximation to the solution of (1) and $\mathbf{f}^{(j)}$ the error function corresponding to $\mathbf{f}\left(\mathbf{x}_{f}^{(j)}\right)$. The next vector of the iterative process $\mathbf{x}_{f}^{(j+1)}$ is obtained by a quasi-Newton iteration according to

$$
\mathbf{x}_{f}^{(j+1)}=\mathbf{x}_{f}^{(j)}+\mathbf{h}^{(j)}
$$

where $\mathbf{h}^{(j)}$ is given by:

$$
\mathbf{h}^{(j)}=-\left(\mathbf{B}^{(j)}\right)^{-1} \mathbf{f}^{(j)}
$$

and $\mathbf{B}^{(j)}$ is an approach to the Broyden matrix [29]:

$$
\mathbf{B}^{(j+1)}=\mathbf{B}^{(j)}+\frac{\mathbf{f}^{(j+1)} \mathbf{h}^{(j) T}}{\mathbf{h}^{(j) T} \mathbf{h}^{(j)}}
$$

which is also updated at each iterative step. In (4), $\mathbf{f}^{(j+1)}$ is obtained by evaluating (1) using a certain parameter extraction method providing the coarse model parameters from the fine model parameters, and the super-index $T$ stands for transpose.

\subsection{Application of ASM to the synthesis of CSRR- and OCSRR-loaded lines.}

To start the ASM, we first need an initial geometry for the structure. Then, the circuit parameters are extracted from the electromagnetic simulation of the initial layout, we compute the error function, and initiate the Broyden matrix. Then the process is iterated until convergence is achieved. For the CSRR-loaded lines, the parameter extraction method, generation of the first layout, and initiation of the Broyden matrix has been explained in detail in [30]. To illustrate the possibilities of the approach, we report the synthesis of a CSRR-loaded line with the circuit elements indicated in table 1. Application of the developed ASM algorithm has lead to the geometry given in table 2 , and convergence (the error is indicated in the table) has been 
achieved after a single iteration (the reason is that we have applied a pre-optimization, also described in [30], so that the initial layout is already very close to the target).

Table 1. Circuit elements of the CSRR-loaded line

\begin{tabular}{rrrr}
\hline$L(\mathrm{nH})$ & $C(\mathrm{pF})$ & $L_{c}(\mathrm{nH})$ & $C_{c}(\mathrm{pF})$ \\
\hline 4.860 & 1.880 & 2.417 & 3.053
\end{tabular}

Table 2. Geometry of the synthesized structure

\begin{tabular}{ccccc}
\hline$l(\mathrm{~mm})$ & $W(\mathrm{~mm})$ & $c(\mathrm{~mm})$ & $d(\mathrm{~mm})$ & Error \\
\hline 8.47 & 2.22 & 0.36 & 0.17 & $<1 \%$
\end{tabular}

Figure 3 depicts the target frequency response, i.e., the one resulting from the circuit simulation of the circuit of Fig. 1(b) with the elements of table 1, compared to the electromagnetic simulation of the initial and final layout. As can be seen the initial layout EM simulation is already very close to the target, and the difference between the target response and that of the final layout is roughly undistinguishable.
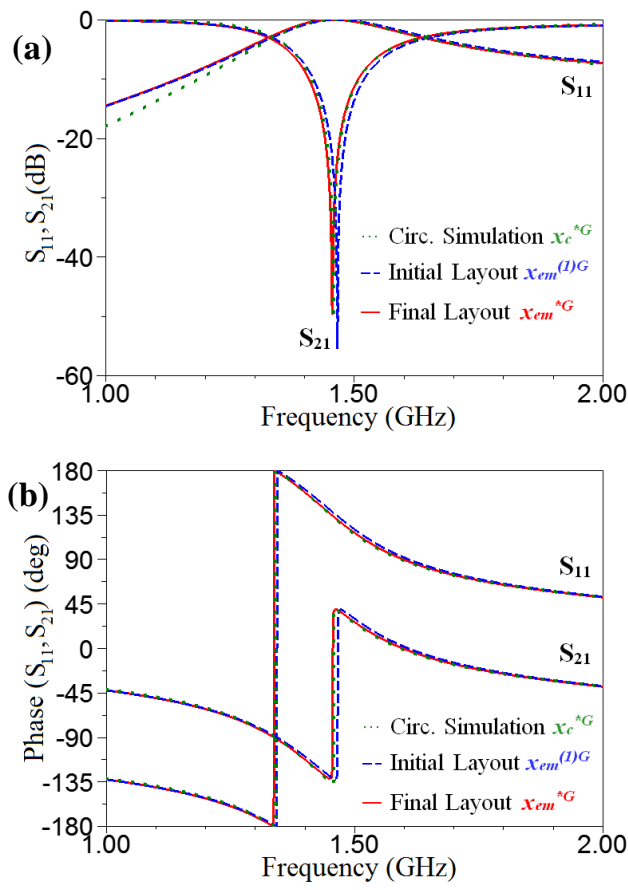

Figure 3: Magnitude (a) and phase (b) of the scattering parameters $S_{21}$ and $S_{11}$ at initial solution $\boldsymbol{x}_{e m}{ }^{(\mathbf{1})}$, final solution $\boldsymbol{x}_{\boldsymbol{e} m}{ }^{*}$, and circuit simulation of $\boldsymbol{x}_{\boldsymbol{c}}{ }^{*}$ (the parameters of table 1). The considered substrate in the (lossless) EM simulation is the Rogers RO3010 with thickness $\mathrm{h}=1.27 \mathrm{~mm}$ and dielectric constant $\mathcal{E}_{r}=10.2$.

For the OCSRR-loaded microstrip lines, the procedure to determine the layout is similar, but there are some differences that must be explained in detail (we dedicate further effort to the synthesis of this particle since the details of the CSRR-loaded line were provided in [30]). First of all, the geometrical variables in the OCSRR-loaded structure of Fig. 2(a) are not the same ones than in Fig. 1(a). Notice that, as mentioned before, the access lines are not considered. However, there is a strip connecting the ports with the inner metallic region of the OCSRR. Thus we have 4 geometrical parameters in the $\operatorname{OCSRR}\left(c, d, r_{e x t}\right.$ and $e$ ), indicated in Fig. 2(a). Since the number of electrical parameters of the circuit model (Fig. 2b) is 3, and it is convenient to deal with the same number of parameters in both spaces (optimization and validation spaces), one of the geometrical parameters is set to a fixed value (this restriction will be relaxed later, in a second ASM optimization of the OCSRR - see sections 4 and 5). Specifically, the slot width is set to $c=0.25 \mathrm{~mm}$.

To determine the initial OCSRR geometry, necessary to initiate the Broyden matrix, the model of the CSRR reported in [22] is considered. The use of this model is justified since, to a first order approximation, the capacitance of the OCSRR $\left(C_{p}\right)$ coincides with the capacitance of the CSRR $\left(C_{c}\right)$, and the inductance of the OCSRR $\left(L_{p}\right)$ is four times larger than the inductance of the CSRR $\left(L_{c}\right)$. This is true as far as $c, d$ and $r_{e x t}$ are identical for both particles and they are etched on the same substrate [31]. On the other hand, to estimate $e$, well-known formulas providing the inductance of an electrically small and narrow strip section are used. Once the initial layout is known, the Broyden matrix is obtained by slightly perturbing OCSRR dimensions $\left(d, r_{e x t}\right.$ and $\left.e\right)$ and obtaining the values of $C_{p}, L_{p}$ and $L_{s h}$ from parameter extraction. This allows us to compute the initial Broyden matrix as follows:

$$
\mathbf{B}^{(1)}=\left(\begin{array}{lll}
\frac{\delta L_{p}}{\delta r_{e x t}} & \frac{\delta L_{p}}{\delta d} & \frac{\delta L_{p}}{\delta e} \\
\frac{\delta C_{p}}{\delta r_{e x t}} & \frac{\delta C_{p}}{\delta d} & \frac{\delta C_{p}}{\delta e} \\
\frac{\delta L_{s h}}{\delta r_{e x t}} & \frac{\delta L_{s h}}{\delta d} & \frac{\delta L_{s h}}{\delta e}
\end{array}\right)
$$

Once the Broyden matrix is initiated, the algorithm can be iterated using (2), where the first error function in (3) is inferred from the extracted parameters of the first layout.

Concerning parameter extraction from the electromagnetic simulation results of a given layout, the three conditions considered are: (i) the position of the transmission zero frequency:

$$
f_{z}=\frac{1}{2 \pi} \sqrt{\frac{1}{C_{p}}\left(\frac{1}{L_{p}}+\frac{1}{L_{s h}}\right)}
$$

(ii) the position of the reflection zero frequency (or intrinsic resonance of the OCSRR):

$$
f_{o}=\frac{1}{2 \pi} \sqrt{\frac{1}{L_{p} C_{p}}}
$$

and (iii) the susceptance slope at $f_{\mathrm{o}}$. All these parameters can be easily inferred from the electromagnetic simulation.

As a synthesis example, we report here the automated layout generation of an OCSRR with target values indicated in table 3 .

Table 3. Circuit elements of the OCSRR-loaded line

\begin{tabular}{ccc}
\hline$L_{s h}(\mathrm{nH})$ & $L_{p}(\mathrm{nH})$ & $C_{p}(\mathrm{pF})$ \\
\hline 0.5 & 3.8 & 4.1
\end{tabular}


The considered substrate is the Rogers RO3010 with thickness $h=0.254 \mathrm{~mm}$ and dielectric constant $\varepsilon_{r}=10.2$. The error function (1) is obtained as follows

$$
\left\|\mathbf{f}\left(x_{f}\right)\right\|=\sqrt{\left(1-\frac{L_{p}}{L_{p}{ }^{*}}\right)^{2}+\left(1-\frac{C_{p}}{C_{p}{ }^{*}}\right)^{2}+\left(1-\frac{L_{s h}}{L_{s h}{ }^{*}}\right)^{2}}
$$

and the algorithm ends when (8) is smaller than a certain predefined value. In our case, it has been found that eight iterations suffice to obtain an error function smaller than $1 \%$. The evolution of the error function is depicted in Fig. 4.

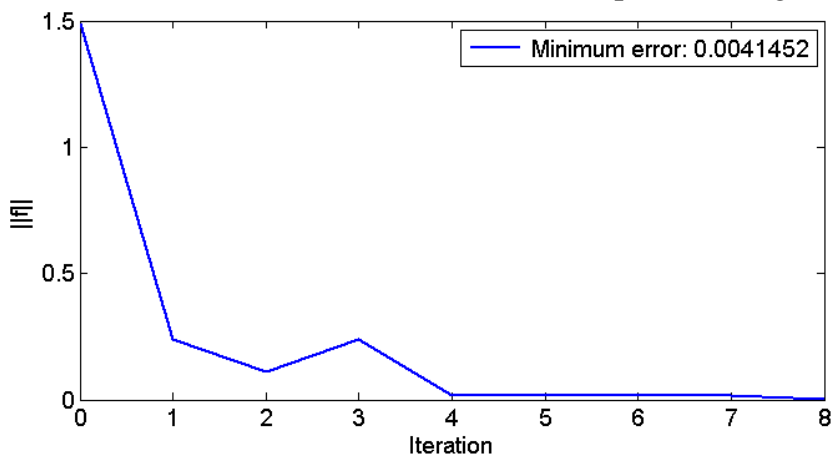

Figure 4: Evolution of the error function with the number of iterations.

The synthesis process has provided the layout depicted in Fig. 5. The geometrical variables are indicated in table 4 . The target response, namely, the one that results by circuit simulation of the circuit of Fig. 2(b) with the target values, and the response obtained from (lossless) electromagnetic simulation of the generated layout, are depicted in Fig. 6. As can be seen, the agreement between the circuit (target) and electromagnetic simulation after optimization is excellent in the frequency range shown, covering a span beyond three times the OCSRR intrinsic resonance frequency. However, notice that the electromagnetic simulation of the first (initial) layout, also depicted in Fig. 6, is very different, pointing out the need for the proposed synthesis algorithm.

Table 4. Geometry of the synthesized OCSRR

\begin{tabular}{ccccc}
\hline$e(\mathrm{~mm})$ & $r_{\text {ext }}(\mathrm{mm})$ & $c(\mathrm{~mm})$ & $d(\mathrm{~mm})$ & Error \\
\hline 0.52 & 2.25 & 0.25 & 0.35 & $<1 \%$
\end{tabular}

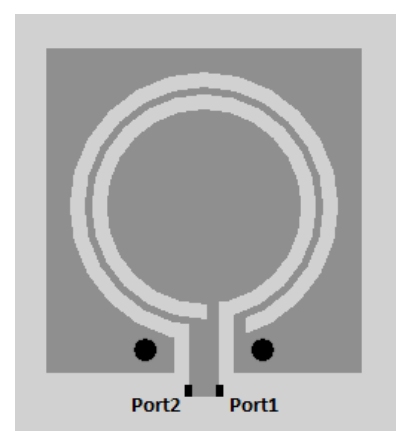

Figure 5: Final layout of the synthesized OCSRR. Dimensions are those indicated in table 4 (the ground plane is indicated in light grey).
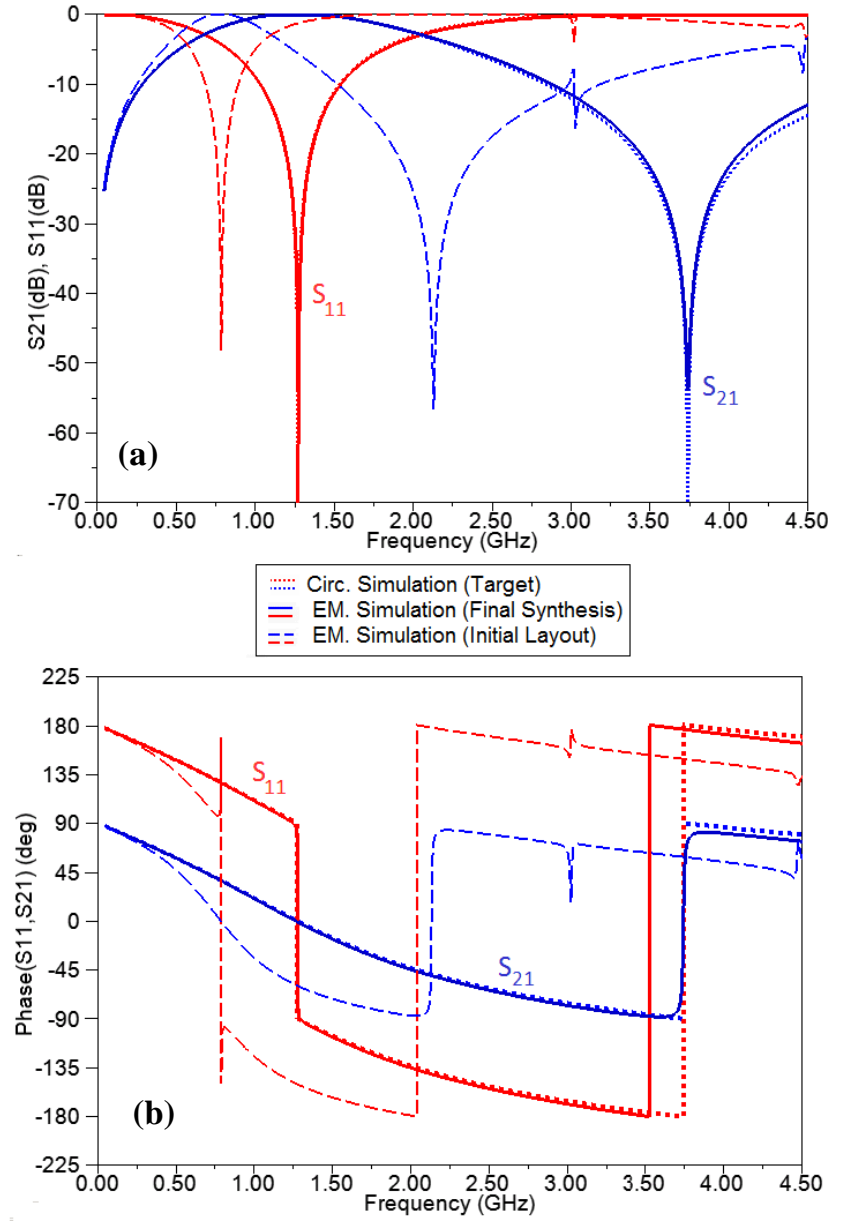

Figure 6: Comparison of the target response and electromagnetic simulation of the generated layout. (a) Magnitude; (b) Phase. The electromagnetic simulation of the initial geometry is also included for comparison purposes.

\section{Discussion on the synthesis of OCSRRs}

In the preceding section, the synthesis of OCSRRs has been realized by setting the value of the slot distance to $c=$ $0.25 \mathrm{~mm}$. In this way, the number of geometrical and electrical variables involved in the synthesis algorithm is the same. Nevertheless, with this approach the range of implementable values of $L_{p}, C_{p}$ and $L_{s h}$ may be limited. Therefore, another potential approach, where none of the geometrical variables $\left(c, d, r_{e x t}\right.$ and $e$ ) is set to a fixed value, is discussed next.

The idea consists on determining regions in the $L_{p}-C_{p}$ space corresponding to implementable values of these variables for different values of the external radius $r_{\text {ext }}$, and considering the target value of $L_{s h}$. The procedure is as follows. First of all, we set $r_{\mathrm{ext}}$ to a certain reasonable value, and $c$ and $d$ to the minimum value of the available technology $\left(c_{\min }, d_{\min }\right)$, and we optimize $e$ (by means of a specific one-variable aggressive space mapping algorithm) in order to recover the target value of $L_{s h}$. From the resulting layout, we obtain through parameter extraction the values of $L_{p}$ and $C_{p}$, corresponding to a point in the $L_{p}-C_{p}$ space. We repeat this procedure by maintaining $r_{e x t}$ to the same value, 
and setting $c=c_{\min }$ and $d=d_{\max }$ (the maximum allowed inter-slot distance). This gives another point in the $L_{p}-C_{p}$ space. Next, we repeat the process for $c=c_{\max }$ and $d=d_{\min }$, and for $c=c_{\max }$ and $d=d_{\max }$, giving two additional points in the $L_{p}-C_{p}$ space. Thus, the four points define a polygon in the plane $L_{p}-C_{p}$. It is expected that if the target value of $L_{p}$ and $C_{p}$ corresponds to a point within that polygon, then there will exist a solution for $c$ and $d$ (with the considered value of $r_{\text {ext }}$ ) satisfying $c_{\text {min }}<c<c_{\max }$ and $d_{\min }<d<d_{\max }$, and providing the required (target) values of $L_{p}$ and $C_{p}$. Notice that with this procedure, we obtain a polygon in the plane $L_{p}-C_{p}$ for a certain value of $r_{e x t}$. The next step is to repeat this process for a different value of $r_{e x t}$. Obviously, the larger the external radius $r_{e x t}$, the larger the values of $L_{p}$ and $C_{p}$ at the four vertexes of the polygon. Thus, the criterion to determine the external radius $r_{e x t}$ is simply to choose the smallest value that provides a polygon enclosing the target value of $L_{p}$ and $C_{p}$ (optimum polygon). This procedure optimizes the size of the OCSRR. If the ratio between the target $L_{p}$ and $C_{p}$ values is too extreme, it is possible that none of the polygons contains the point associated to such pair of element values. In that case, the resonator is not synthesizable with an OCSRR.

Assuming that the target values $L_{p}{ }^{*}, C_{p}{ }^{*}$ and $L_{s h}{ }^{*}$ can be physically implemented, $r_{e x t}$ is determined through the procedure detailed in the previous paragraph. A further step is required to obtain the initial values of $c, d$ and $e$ necessary to initiate the ASM algorithm, unless $L_{p}{ }^{*}, C_{p}{ }^{*}$ coincide with any of the vertices of the smaller polygon enclosing the $L_{p}{ }^{*}$ $C_{p}{ }^{*}$ point (in this case, the layout is already known, and, hence, no further optimization is necessary). A similar procedure to that reported in [30] for CSRR-loaded lines can be applied to determine the initial values of $c, d$ and $e$ (rather than determining them from existing, but not accurate enough, models, as reported in the preceding section). It is expected that the dimensions of the OCSRR after ASM optimization depend on the position of the point $L_{p}{ }^{*}, C_{p}{ }^{*}$ in the optimum polygon. Namely, if the point $L_{p}{ }^{*}, C_{p}{ }^{*}$ is close to a vertex, it is expected that $c, d$ and $e$ are similar to the values corresponding to that vertex.

The specific procedure to determine the initial layout ( $c$, $d$ and $e$ ) is as follows [30]: we assume that $c, d$ and $e$ have a linear dependence on $L_{p}$ and $C_{p}$. In particular, for $c$ we can write (identical expressions are used for the other variables):

$$
c=f\left(L_{p}, C_{p}\right)=\left(A+B L_{p}\right)\left(C+D C_{p}\right)
$$

The previous expression can be alternatively written as

$$
c=f\left(L_{p}, C_{p}\right)=a_{0}+a_{1} L_{p}+a_{2} C_{p}+a_{3} L_{p} C_{p}
$$

where the constants $a_{i}$ determine the functional dependence of the initial value of $c$ with $L_{p}$ and $C_{p}$. To determine the constants, four conditions are needed. Let us consider the following error function:

$$
f_{\text {error }}=\sum_{j=1}^{N_{v}}\left(c_{j}-f\left(L_{p_{j}}, C_{p_{j}}\right)\right)^{2}
$$

where the subscript $j$ is used to differentiate between the different vertices, and hence $c_{j}$ is the value of $c$ in the vertex $j$, and $L_{p j}$ and $C_{p j}$ are the corresponding values of $L_{p}$ and $C_{p}$ for that vertex. We have considered a number of vertices equal to $N_{v}=4$ (as mentioned before), but the formulation can be extended to a higher number of vertices. The previous error function can be written as

$$
\begin{gathered}
f_{\text {error }}=\sum_{j=1}^{4} c_{j}^{2}-2 \sum_{j=1}^{4} c_{j} \cdot\left(a_{0}+a_{1} L_{p_{j}}+a_{2} C_{p_{j}}+a_{3} L_{p_{j}} C_{p_{j}}\right)+ \\
+\sum_{j=1}^{4}\left(a_{0}+a_{1} L_{p_{j}}+a_{2} C_{p_{j}}+a_{3} L_{p_{j}} C_{p_{j}}\right)^{2}
\end{gathered}
$$

To find the values of the constants $a_{i}$, we obtain the partial derivatives of the previous error function with regard to $a_{i}$ and force them to be equal to zero [32] as

$$
\begin{gathered}
\frac{\partial f_{\text {error }}}{\partial a_{i}}=-2 \sum_{j=1}^{4} c_{j} \frac{\partial f\left(L_{p_{j}}, C_{p_{j}}\right)}{\partial a_{i}}+ \\
+2 \sum_{j=1}^{4}\left[f\left(L_{p_{j}}, C_{p_{j}}\right) \frac{\partial f\left(L_{p_{j}}, C_{p_{j}}\right)}{\partial a_{i}}\right]=0
\end{gathered}
$$

for $i=1,2,3,4$. Following this least-squares approach, four independent equations for the constants $a_{i}$ are obtained. Such equations can be written in matrix form as:

$$
\left(\begin{array}{cccc}
4 & \sum_{j=1}^{4} L_{p_{j}} & \sum_{j=1}^{4} C_{p_{j}} & \sum_{j=1}^{4} L_{p_{j}} C_{p_{j}} \\
\sum_{j=1}^{4} L_{p_{j}} & \sum_{j=1}^{4} L_{p_{j}}^{2} & \sum_{j=1}^{4} L_{p_{j}} C_{p_{j}} & \sum_{j=1}^{4} L_{p_{j}}^{2} C_{p_{j}} \\
\sum_{j=1}^{4} C_{p_{j}} & \sum_{j=1}^{4} L_{p_{j}} C_{p_{j}} & \sum_{j=1}^{4} C_{p_{j}}^{2} & \sum_{j=1}^{4} C_{p_{j}}^{2} L_{p_{j}} \\
\sum_{j=1}^{4} L_{p_{j}} C_{p_{j}} & \sum_{j=1}^{4} L_{p_{j}}^{2} C_{p_{j}} \sum_{j=1}^{4} C_{p_{j}}^{2} L_{p_{j}} & \sum_{j=1}^{4} C_{p_{j}}^{2} L_{p_{j}}^{2}
\end{array}\right)\left(\begin{array}{l}
a_{o} \\
a_{1} \\
a_{2} \\
a_{3}
\end{array}\right)=
$$

Once the constants are obtained (solving the previous equations), the initial value of $c$ is inferred from (10). The process is repeated for $d$ and $e$, and the initial geometry necessary for the initiation of the ASM algorithm is thus obtained.

To gain insight on the previous procedure to determine the initial layout of the OCSRR, where none of the geometrical variables is a priori fixed, various polygons in the $L_{p}-C_{p}$ plane (for different radius) have been calculated. Namely, we have set $e$ to $0.5 \mathrm{~mm}$ (this gives approximately the same value $L_{s h}$ ), and we have extracted the pair of values $L_{p}$ and $C_{p}$ for the extreme combinations of $c$ and $d$ considering different external radius, $r_{\text {ext }}$. The results are depicted in Fig. 7. As expected the polygons roughly scale 
with $r_{\text {ext }}$, since $L_{p}$ and $C_{p}$ are approximately proportional to $r_{e x t}$ for a fixed value of $c$ and $d$. Notice that the polygons define a pair of roughly diagonal envelopes in the $L_{p}-C_{p}$ plane. The region contained between both envelopes is the region of implementable values of $L_{p}$ and $C_{p}$ for the considered value of $L_{s h}$. The results also confirm that there is an optimum external radius for the OCSRR, i.e., the smaller one whose corresponding polygon encloses the target values of $L_{p}$ and $C_{p}$.

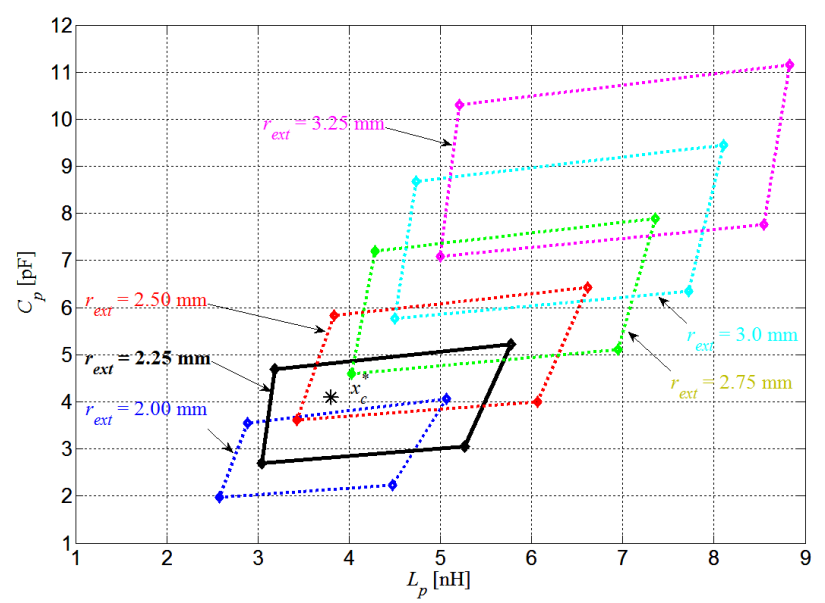

Figure 7: Polygons in the $L_{p}-C_{p}$ space corresponding to the indicated values of the external radius. The four combinations for each polygon correspond to extreme values of $c$ and $d$, that is, $c=c_{\text {min }}, d=d_{\text {min }}$; $c=c_{\text {min }}, d=$ $d_{\max } ; c=c_{\max }, d=d_{\min } ; c=c_{\max }, d=d_{\max }$, with $c_{\min }=d_{\min }=0.15 \mathrm{~mm}$ and $c_{\max }=d_{\max }=0.45 \mathrm{~mm}$. In all the cases $e=0.5 \mathrm{~mm}$ corresponding to an inductance of approximately $L_{s h}=0.5 \mathrm{nH}$. The optimum radius for the considered target value of $L_{p}$ and $C_{p}$ (indicated by the symbol *) is $r_{e x t}=2.25 \mathrm{~mm}$, and the corresponding polygon is highlighted.

Thus, the alternative approach for the determination of the initial layout of the OCSRR, rather than forcing one of the geometrical variables to have a certain value, it determines the optimum radius, and then gives the initial values of $c, d$ and $e$ (to be optimized in the ASM process) from a least squares approach (rather than from existing, but inaccurate, models). The application of this strategy for the synthesis of OCSRRs has been also developed (a synthesis example is reported in the next section).

\section{Synthesis of an OCSRR-loaded line by means of the optimized method}

Specifically we have considered the synthesis of an OCSRR with the target parameters indicated in table 3. For the synthesis we have strictly applied the method explained before, i.e., we have determined the polygons for different radius by forcing that the value of $L_{s h}$ at each point of the polygon is the target value. Since the polygons of Fig. 7 were obtained by considering a fixed value of $e$ (this speeds the calculation since a space mapping is not required), we have not considered the radius of the highlighted polygon (in spite that the final radius is expected to be similar).

To determine the radius, we have obtained the polygons corresponding to two extreme radius $\left(r_{e x t}=2 \mathrm{~mm}\right.$ and $r_{\text {ext }}=$ $3.25 \mathrm{~mm}$ ), and we have calculated the distance between the target point and the diagonals given by the crossed points $\left[c_{\min }, d_{\max }\right]$ and $\left[c_{\max }, d_{\min }\right]$ of both polygons. By interpolation we have estimated the optimum radius, which has been found to be $r_{\text {ext }}=2.198 \mathrm{~mm}$. The polygon corresponding to such radius is depicted in Fig. 8, together with the target value of $L_{p}$ and $C_{p}$. The value of $e$ in each vertex gives the target value of $L_{s h}$, and has been calculated by means of a specific one-variable ASM, as indicated before. Notice that the radius can indeed be further optimized since the target point is very close to the diagonal of the polygon (indicated in Fig. 8), rather than to the upper side. Nevertheless, the calculated radius is expected to be very close to the most optimum one, and is considered to be the optimum radius in this work (further optimization is left for a future work).

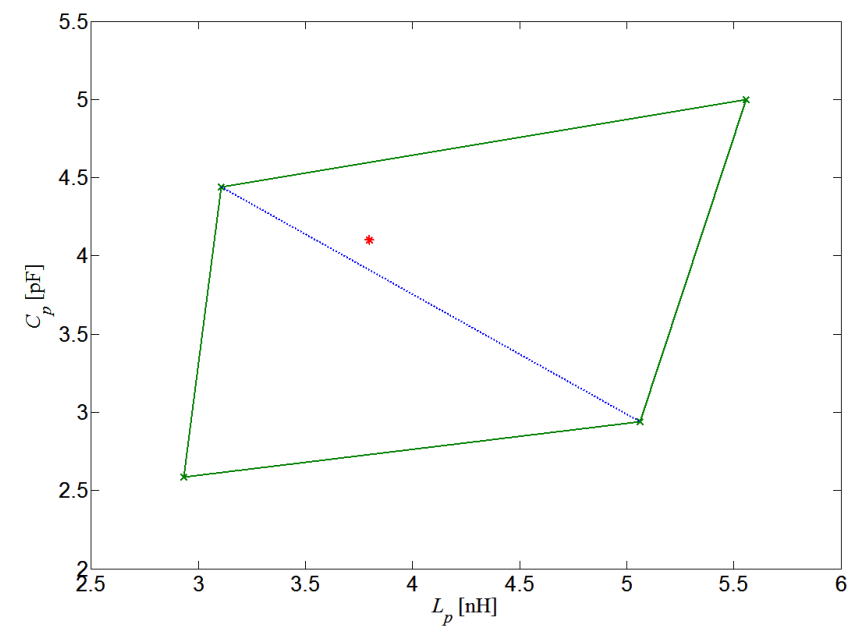

Figure 8: Polygon in the $L_{p}-C_{p}$ space corresponding to the optimum value of the external radius, calculated according to the indicated procedure.

Once the radius and the associated polygon have been determined, we apply the least squares approach presented before for the determination of the first layout, namely $c, d$ and $e$. The values of these geometrical variables have been found to be $c=0.229 \mathrm{~mm}, d=0.353 \mathrm{~mm}$, and $e=0.461 \mathrm{~mm}$. Application of the second ASM has been found to provide an error function smaller than $1 \%$ in only one iteration step. The final geometry is given in table 5 . The circuit simulation with the target values and the electromagnetic simulation of the generated layout are compared in Fig. 9. Again, the agreement is excellent, pointing out the validity of this alternative synthesis method. As compared to the OCSRR synthesis reported in section 3, with identical target elements, the generated geometry with this new method provides smaller radius, $c$ and $d$, and hence a smaller OCSRR size.

Table 5. Geometry of the synthesized OCSRR using the second approach

\begin{tabular}{ccccc}
\hline$e(\mathrm{~mm})$ & $r_{\text {ext }}(\mathrm{mm})$ & $c(\mathrm{~mm})$ & $d(\mathrm{~mm})$ & Error \\
\hline 0.474 & 2.198 & 0.215 & 0.339 & $<1 \%$
\end{tabular}




\section{Conclusions}

In conclusion, we have applied ASM optimization to the synthesis of CSRR- and OCSRR-loaded microstrip lines, with special emphasis in OCSRR loaded lines. The model of a shunt connected OCSRR in microstrip technology, including the series inductance, has been considered. With this model, the OCSRR is accurately described up to frequencies much beyond its resonance frequency, and the transmission zero, typical of these particles in shunt connection, can be predicted.
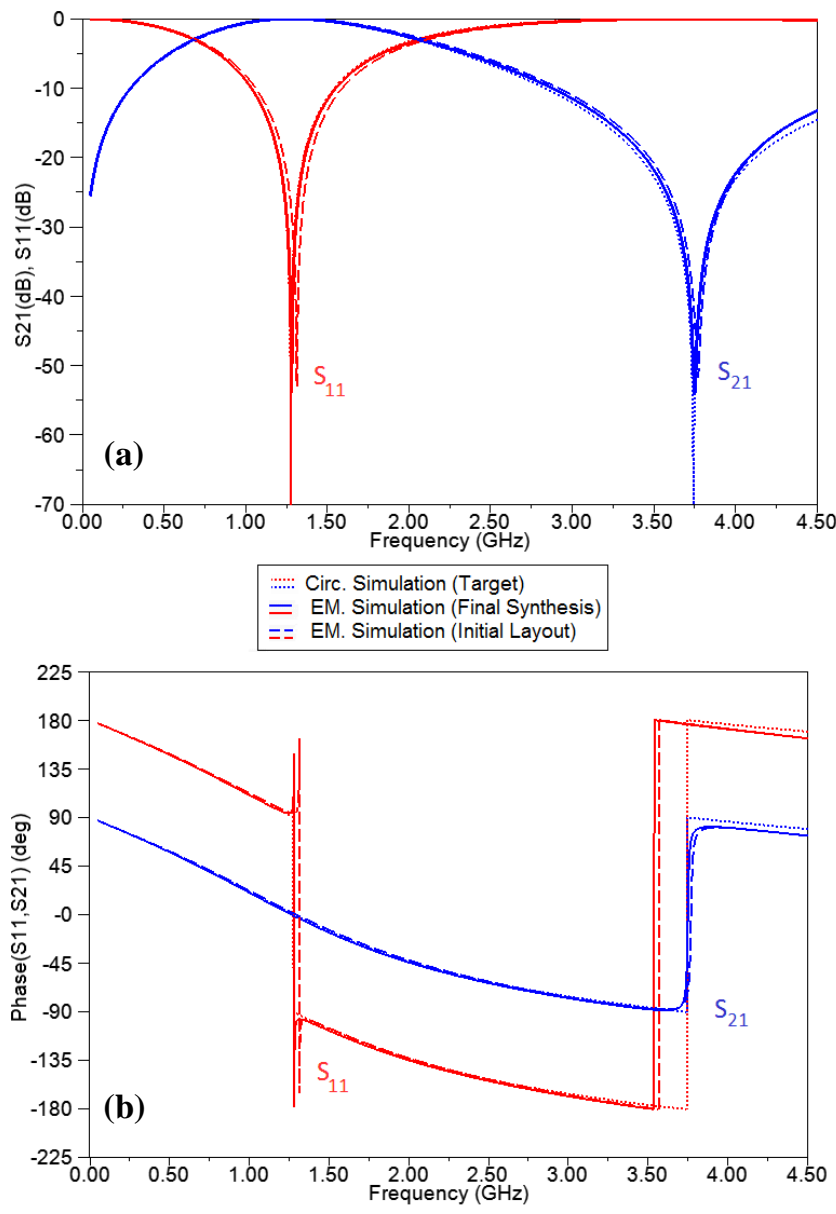

Figure 9: Comparison of the target response and electromagnetic simulation of the generated layout. (a) Magnitude; (b) Phase. The electromagnetic simulation of the initial geometry is also included for comparison purposes.

We have considered two alternative OCSRR optimization schemes. In the first one, the optimization variables have been considered to be the external radius, $r_{e x t}$, the distance between the slots, $d$, and the width of the metallic strip, $e$, connecting the host line and the inner metallic region of the OCSRR. The width of the slots, $c$, has been forced to a certain reasonable value. With this strategy, the number of geometrical variables is the same than the number of electrical variables of the circuit model, being this very convenient in ASM optimization. Concerning the initial layout, necessary to initiate the ASM synthesis process, existing models linking electrical variables and geometry for both the OCSRR and the inductive strip have been considered. We have applied this optimization approach to the synthesis of an OCSRR. The generated layout has been simulated, and the electromagnetic response has been found to be in very good agreement with the circuit simulation of the electrical model, thus validating the model and the synthesis method.

In the second optimization approach, fixing one geometrical variable of the OCSRR to a certain value has been avoided. With this approach, the optimum external radius that minimizes particle size is determined, and the synthesis process involves the other three geometrical variables. A method to determine the initial layout, based on least squares, has been pointed out. This alternative method provides smaller particle size, as compared to the first optimization technique, whilst the time to convergence is comparable, in spite that a pre-ASM is required to determine the convergence region in the coarse subspace.

In summary, the results of this paper confirm that the proposed ASM techniques are efficient and useful for the synthesis of CSRRs and their open counterparts, OCSRRs. Moreover, it is clear that for OCSRRs the three elements of the equivalent circuit model do not univocally determine the layout of the particle. Many solutions exist as long as the geometry is determined by four parameters. However, we have proposed a synthesis technique that reduces particle size.

\section{Acknowledgements}

This work has been partially supported by MICIIN-Spain (projects TEC2010-17512 METATRANSFER, TEC201021520-C04-01 AVANSAT, CONSOLIDER EMET CSD2008-00066, and grant AP2008-04707), Generalitat de Catalunya (project 2009SGR-421), and MITyC-Spain (project TSI-020100-2010-169 METASINTESIS). Ferran Martín is in debt to ICREA for supporting his work through an ICREA Academia Award (calls 2008 and 2013).

\section{References}

[1] G.V. Eleftheriades and K.G. Balmain, Negative refraction metamaterials: fundamental principles and applications, John Wiley \& Sons, Inc, New Jersey 2005.

[2] C. Caloz and T. Itoh, Electromagnetic Metamaterials: Transmission Line Theory and Microwave Applications, John Wiley \& Sons, 2006.

[3] R. Marqués, F. Martín and M. Sorolla, Metamaterials with negative parameters: theory, design and microwave applications, John Wiley \& Sons, Inc, New Jersey 2008.

[4] F. Martín, Artificial Transmission Lines for $R F$ and Microwave applications, John Wiley \& Sons, Inc, New Jersey, to be published.

[5] M.A. Antoniades and G.V. Eleftheriades, "A broadband series power divider using zero-degree metamaterial 
phase shifting lines", IEEE Microwave and Wireless Components Letters, vol. 15, pp. 808-810, Nov. 2005.

[6] H. Okabe, C. Caloz and T. Itoh, "A compact enhanced bandwidth hybrid ring using an artificial lumped element left handed transmission line section", IEEE Trans. Microwave Theory and Techniques, vol. 52, pp. 798-804, March 2004.

[7] G. Sisó, J. Bonache, M. Gil and F. Martín, “Application of resonant-type metamaterial transmission lines to the design of enhanced bandwidth components with compact dimensions", Microwave and Optical Technology Letters, vol. 50, pp. 127-134, January 2008.

[8] I.H. Lin, M. De Vincentis, C. Caloz and T. Itoh, "Arbitrary dual-band components using composite right/left handed transmission lines", IEEE Trans. Microwave Theory and Techniques, vol. 52, pp. 11421149, April 2004.

[9] A. C. Papanastasiou, G. E. Georghiou, and G. V. Eleftheriades, "A quad-band wilkinson power divider using generalized NRI transmission lines", IEEE Microwave and Wireless Components Letters, vol. 18, pp. 521-523, August 2008.

[10] M. Durán-Sindreu, G. Sisó, J. Bonache and F. Martín, "Planar multi-band microwave components based on the generalized composite right/left handed transmission line concept", IEEE Transactions on Microwave Theory and Techniques, vol. 58, no 12, pp. 3882-3891, Dec. 2010.

[11] J. Bonache, I. Gil, J. García-García, F. Martín, "Novel microstrip band pass filters based on complementary split ring resonators", IEEE Transactions on Microwave Theory and Techniques, vol. 54, pp. 265-271, January 2006.

[12] M. Gil, J. Bonache, J. García-García, J. Martel and F. Martín, "Composite right/left handed (CRLH) metamaterial transmission lines based on complementary split rings resonators (CSRRs) and their applications to very wide band and compact filter design", IEEE Transactions on Microwave Theory and Techniques, vol. 55, pp. 1296-1304, June 2007.

[13] S. Lim, C. Caloz, and T. Itoh, "Metamaterial-based electronically-controlled transmission line structure as a novel leaky-wave antenna with tunable angle and beamwidth," IEEE Trans. Microwave Theory Techniques, vol. 52, No. 12, pp. 2678-2690, December 2004.

[14] G. Zamora, S. Zuffanelli, F. Paredes, F. Javier HerraizMartínez, F. Martín, and J. Bonache, "Fundamental mode leaky-wave-antenna (LWA) using slot line and split-ring-resonator (SRR) based metamaterials", IEEE Antennas and Wireless Propagation Letters, vol. 12, pp. 1424-1427, 2013.

[15] A. K. Iyer and G. V. Eleftheriades. "Negative refractive index metamaterials supporting 2-D waves," in IEEEMTT Int'l Microwave Symp., vol. 2, Seattle, WA, pp. 412-415, June 2002.
[16] A.A. Oliner, "A periodic-structure negative-refractiveindex medium without resonant elements," in URSI Digest, IEEE-AP-S USNC/URSI National Radio Science Meeting, San Antonio, TX, pp. 41, June 2002.

[17] C. Caloz and T. Itoh. "Application of the transmission line theory of left-handed ( $\mathrm{LH})$ materials to the realization of a microstrip LH transmission line," in Proc. IEEE-AP-S USNC/URSI National Radio Science Meeting, vol. 2, San Antonio, TX, pp. 412-415, June 2002.

[18]F. Martín, F. Falcone, J. Bonache, R. Marqués and M. Sorolla, "Split ring resonator based left handed coplanar waveguide", Appl. Phys. Lett., vol. 83, pp. 4652-4654, December 2003.

[19]F. Falcone, T. Lopetegi, M.A.G. Laso, J.D. Baena, J. Bonache, R. Marqués, F. Martín, M. Sorolla, "Babinet principle applied to the design of metasurfaces and metamaterials", Phys. Rev. Lett., vol. 93, paper 197401, November 2004.

[20] M. Durán-Sindreu, A. Vélez, F. Aznar, G. Sisó, J. Bonache and F. Martín, "Application of Open Split Ring Resonators and Open Complementary Split Ring Resonators to the Synthesis of Artificial Transmission Lines and Microwave Passive Components", IEEE Trans. Microwave Theory and Techniques, vol. 57, pp. 3395-3403, Dec. 2009.

[21] A. Vélez, F. Aznar, M. Durán-Sindreu, J. Bonache and F. Martín, "Stop-Band and Band-Pass Filters in Coplanar Waveguide Technology Implemented by Means of Electrically Small Metamaterial-Inspired Open Resonators", IET Microwaves, Antennas and Propagation, vol. 4, pp. 712-716, Jun. 2010.

[22] J.D. Baena, J. Bonache, F. Martín, R. Marqués, F. Falcone, T. Lopetegi, M.A.G. Laso, J. García, I Gil, M. Flores-Portillo and M. Sorolla, "Equivalent circuit models for split ring resonators and complementary split rings resonators coupled to planar transmission lines", IEEE Transactions on Microwave Theory and Techniques, vol. 53, pp. 1451-1461, April 2005.

[23] M. Gil, J. Bonache, J. Selga, J. García-García, F. Martín, "Broadband resonant type metamaterial transmission lines", IEEE Microwave and Wireless Components Letters, vol. 17, pp. 97-99, February 2007.

[24] M. Durán-Sindreu, P. Vélez, J. Bonache and F. Martín, "Broadband Microwave Filters Based on Open Split Ring Resonators (OSRRs) and Open Complementary Split Ring Resonators (OCSRRs): improved models and design optimization", Radioengineering, vol. 20, pp. 775-783, Dec. 2011.

[25] P. Vélez, J. Naqui, M. Durán-Sindreu, J. Bonache, and F. Martín, "Broadband Microstrip Bandpass Filter Based on Open Complementary Split Ring Resonators", International Journal of Antennas and Propagation, vol. 2012, 6 pages, 2012

[26] J.W. Bandler, R.M. Biernacki, S.H. Chen, P.A. Grobelny and R.H. Hemmers, "Space mapping technique for electromagnetic optimization", IEEE 
Trans. Microw. Theory Techn., vol. 42, pp. 2536-2544, Dec. 1994.

[27]J.W. Bandler, R.M. Biernacki, S.H. Chen, R.H. Hemmers, and K. Madsen, "Electromagnetic optimization exploiting aggressive space mapping", IEEE Trans. Microw. Theory Techn., vol. 43, pp. 287425442882, Dec. 1995.

[28] J. W. Bandler, Q. S. Cheng, S. A. Dakroury, A. S. Mohamed, M. H. Bakr, K. Madsen, and J. Søndergaard, "Space mapping: the state of the art," IEEE Trans. Microw. Theory Tech., vol. 52, pp. 337-361, Jan. 2004.

[29]C. G. Broyden, "A Class of Methods for Solving Nonlinear Simultaneous Equations", Mathematics of Computation, vol. 19, no. 92, pp. 577-593, Oct. 1965.

[30] J. Selga, A. Rodríguez, V.E. Boria, and F. Martín, "Synthesis of Split Rings based Artificial Transmission Lines through a New Two-Step, Fast Converging, and Robust Aggressive Space Mapping (ASM) Algorithm”, IEEE Transactions on Microwave Theory and Techniques, vol. 61(6), pp. 2295-2308, June 2013.

[31] A. Velez, F. Aznar, J. Bonache, M.C. VelázquezAhumada, J. Martel and F. Martín, "Open complementary split ring resonators (OCSRRs) and their Application to Wideband CPW Band Pass Filters", IEEE Microwave and Wireless Components Letters, vol. 19, pp. 197-199, April 2009.

[32]D. M. Bates and D. G. Watts, Nonlinear Regression Analysis and Its Applications. New York, NY, USA: Wiley, 1998. 